\title{
A New Approach to Detect Partially Overlapped Coins
}

\author{
Supreet Kaur \\ Computer Science Engineering, \\ Lovely Professional University, Phagwara Punjab, \\ India.
}

\begin{abstract}
Coins are the vital part of our life. It is used at every point whether it is small cafe or in mall. So it's our fundamental need to recognize the coins. Image Processing is a field in which working is done on images. By using this, recognition of different denomination of coins is done. For recognition, first canny edge detection is used to detect edges then Hough Transformation is used to detect coin and then coin partial overlapping is removed. The Hough Transformation is used because it is less prone to noise. GUI will also be created which make system more users friendly and interactive. This can be implemented using MATLAB.
\end{abstract}

KEYWORD:Hough Transform, coin recognition, image processing, morphing, Circular Hough Transform(CHT).

\section{INTRODUCTION}

Images plays very important role in human perception, and vision is the most advanced in human senses. Human is limited to visual band of electromagnetic spectrum but this is not the case with imaging machines, as imaging machines cover almost the entire electromagnetic spectrum(gamma to radio waves)[1]. The images generated by sources can also be operated by this. These include ultrasound, electron microscopy and computer generated images. So, because of above reasons we can develop many applications in various fields.

There are following processes followed for recognition:

1) Low-level process: The process in which the initial operations are performed like image pre-processing which is done to reduce noise, contrast enhancement, image sharpening, etc. Only one thing is focused that input and output must be images.

2) Mid-level process: This type of process perform segmentation (the image is portioned into small objects or regions), description of segmented objects to reduce them so that it will be suitable for computer processing and classification in which recognition of individual object is done. The basic idea behind mid-level is that the input is always image but outputs are the attributes extracted from the input image. These attributes include edges, contours, etc.

3) High-level process: In this process the assembling of recognised objects are considered and performing the cognitive functions normally associated with human vision [1].

The coins are the vital part of our life. They are the oldest and most frequently used legal tender found. They are in usage

\author{
Richa Sharma \\ Assistant Professor, Computer Science, \\ Lovely Professional University, Phagwara Punjab, \\ India
}

since thousands year back. They are in usage from centauries. They are used in buses, trains, shops, etc. Coin recognition is really difficult task because there are $n$ numbers of rotations of it. To mostly challenging work is noise. As, data totally change when noise is present. So, to work upon it Hough Transform is used because it has great capacity to work with noises and also has capacity to extract data from object.

There are different approaches for coin recognition.

1) Text based recognition; the recognition of coin is done through text present on the coin. Text includes country, date of issue, etc. But recognition may be able to be difficult in this as text is blurred after some time span.

2) Content based recognition; in this approach, the recognition is done through image colors, shapes or textures [2]

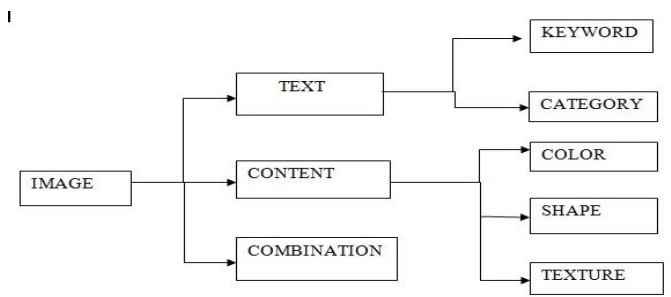

Figure (1): Different approaches for coin recognition.

The polar coordinate image of coin on circles with different radii is used as feature for coin recognition. For the working of image the initial step is pre-processing of image. The preprocessing of image has various stages like cropping, scaling, resizing, and rotation. It is very difficult to work on the original image so many manipulations have to take place. These manipulations are termed as pre-processing. Preprocessing of image includes process termed as zooming and de-zooming. When image is too small the zooming process helps to bigger size of image and it will increase the recognition rate. When one wants to separate bright coin image from darker background or vice versa then usage of thresholding comes to play[3]. Hough Transform is used because of its high reliability and results are appropriate even in presence of noise. This is also time saving approach. Although it has many benefits but still it has drawback, it can't work on large size image [4].

\section{RELATED WORK}

The image is recognised by taking specific areas in searching. It means data covered from various areas is properly evaluated. A smaller size data leads to loss of information and big size data leads to image noise. There are two approaches for rotation normalization. First type of normalization depends 
upon human factor. Human upload pictures but there is a risk that human may make wrong rotation. Second type of normalization is in which machine is used and it may consist of more than one normalized rotation [5].

The image at the top left of Figure shows some objects. In this the information is extract from various sizes of objects. From image objects of interest from the background are separated, this process is termed as segmentation. This usually involves thresholding the image, which is done by setting the values of pixels above a certain threshold value to white, and all the others to black (top right of Figure). As it is uneven data to make it clean filters are applied, this process is termed as image enhancement and restoration as explained above [6].

CHT is used for curve detection. This technique can also detect the object in presence of noise. CHT is the modified version of Hough Transform (HT). The CHT is used to transform a set of feature points in the image space into a set of accumulated votes in a parameter space. Then, for each feature point, votes are accumulated in an accumulator array for all parameter combinations. The array elements that contain the highest number of votes indicate the presence of shape [7].

\section{IMPLEMENTATION}

A digital video camera and a computer are used as a set up. The camera of resolution $320 \mathrm{X} 240$ is taken. The sample image is like image shown in Figure 1.

The coin always resembles a circle whether is fully visible or partial distorted. Circular Hough Transform (CHT) as the capacity to remove noise and identify the coin. Figure 2 represents the flow for detection of coin.

First the image is taken as the input to the system. Then PreProcessing is performed over it. Pre-Processing consist of several steps like zooming, cropping, etc. Then type conversion is taken place as RGB is converted to greyscale or black and white image. This will reduce the size of image. After type conversion, it is checked that whether the coins are partially overlapped or not. If they are partially overlapped then morphing operations are performed like erode or majority. Then the radius of the coin is calculated and the thresholding is adjusted for the detection of the coin. After thresholding adjust, image segmentation and edge detection takes place. It is the most important part as for Hough Transform edge information is needed. And canny edge detection is used because it gives thin edges as compared to sobel and it also fill up the gaps where data is not regular.

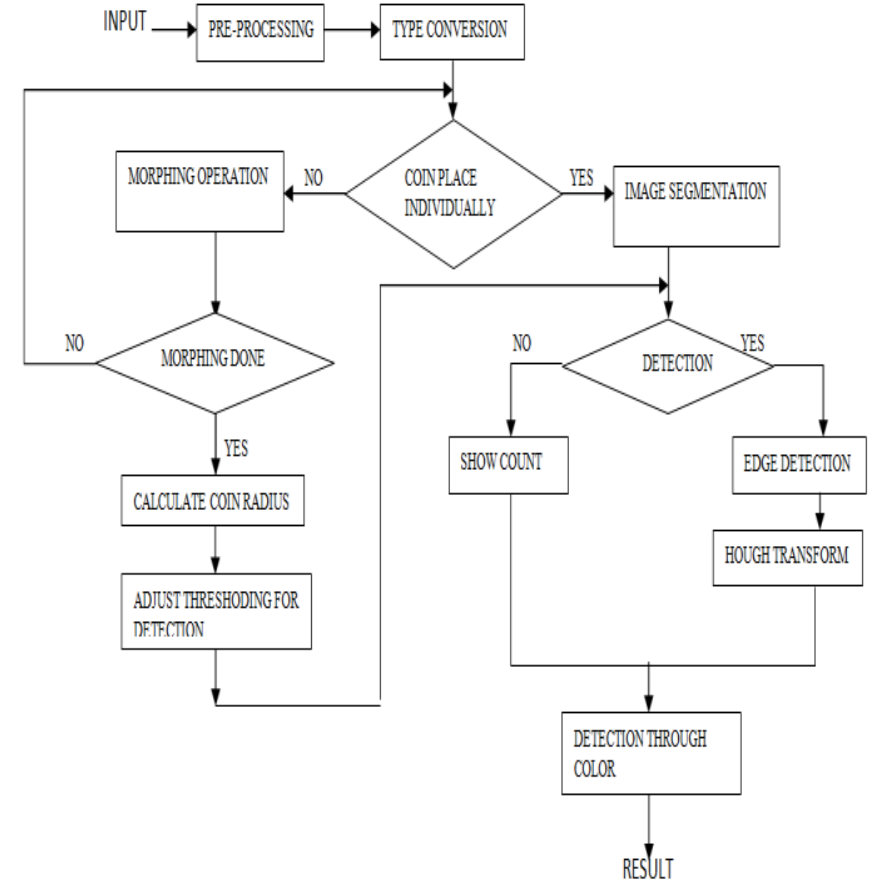

Figure (2): Coin Recognition Process

The equation for the circle is as follow:

$\left(\mathrm{x}-\mathrm{x}_{0}\right)^{2}+\left(\mathrm{y}-\mathrm{y}_{0}\right)^{2}=\mathrm{r}^{2}$

Where, $\mathrm{x}_{0}$ and $\mathrm{y}_{0}$ are the coordinates of the centre of circle and $r$ is the radius of the circle.

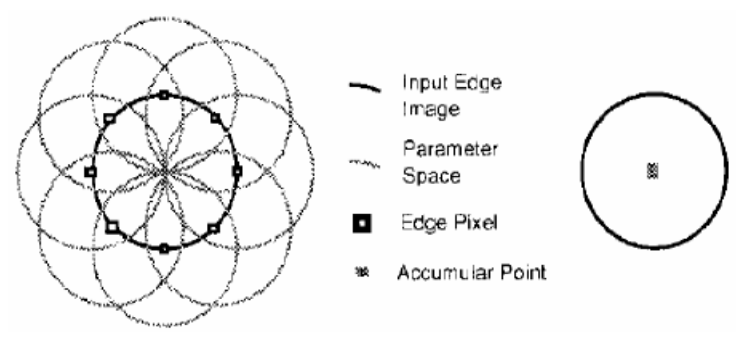

Figure(3): The edge points and corresponding accumulator space.

The edge points in the image are represented by black circles. The circle of the radius $\mathrm{R}$ to an accumulator space is contributed by the edge points. This accumulator space is represented by the grey circles.

\section{EXPERIMENTAL RESULTS}

In figure (4), the detection of individual coin is done. First the original image is read then over it type conversion is done using morphing operation. In this case the detection is done and count of coin is accurate i.e. 6 . 


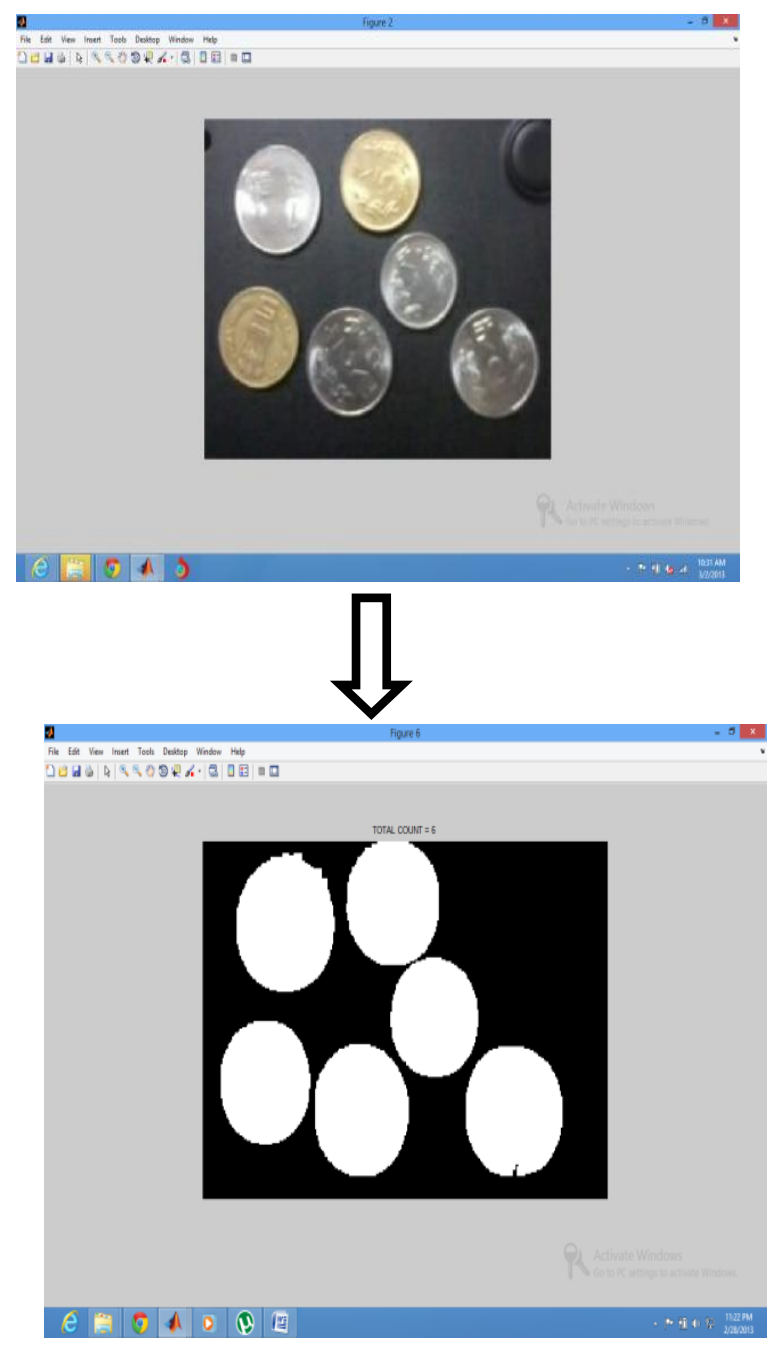

Figure (4): Detection of individual coins

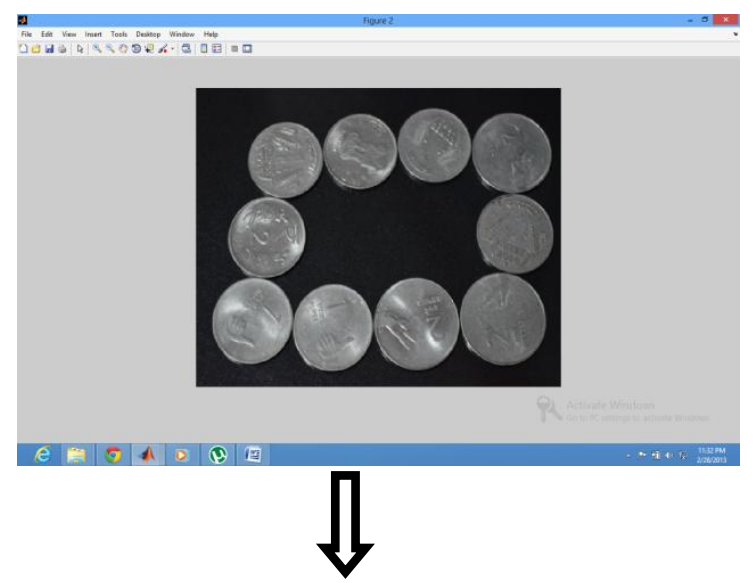

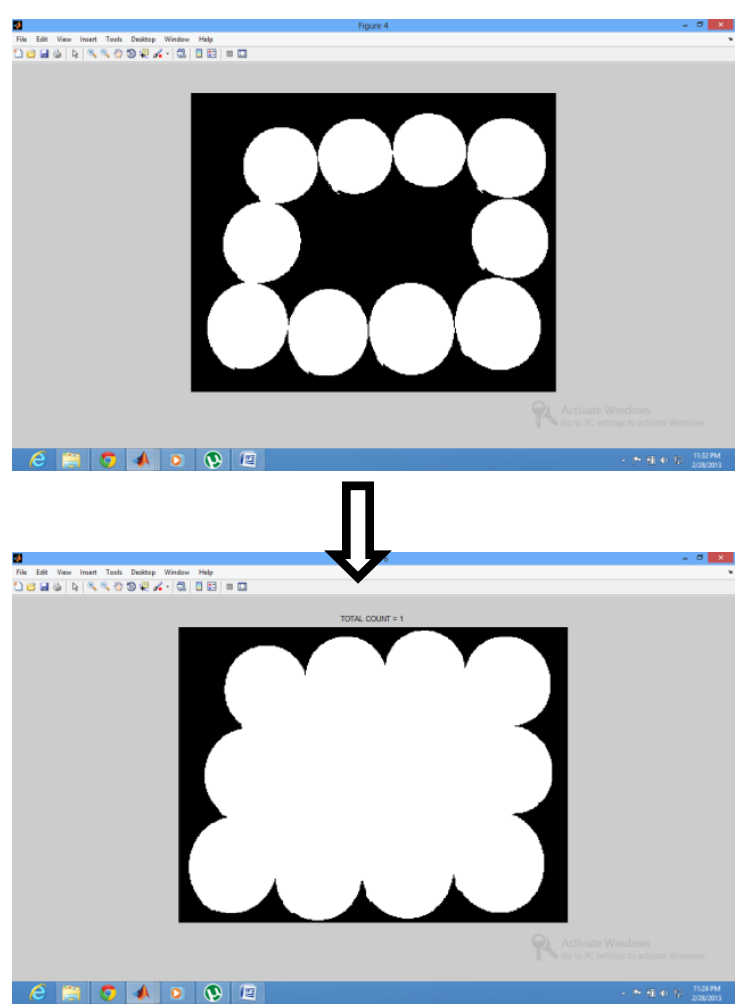

Figure(5): Detection of coins touching each other

In figure (5), the detection of coins touching each other is done. In this first the type conversion is done it converts the original image to its binary image but in this case, detection is not done. Then, morphing operation is done by using "imfill" operator. But by this, the count of coin is detected to be 1 . So, conclusion is that this method works well with individual coins but not with coins touching each other. So, another operation comes to play.

In figure (6), the image taken in figure (5) is again taken. The detection of it is difficult by using "imfill" operation. So, to overcome it the operation known as erode comes to play. By using this operation the edges are compressed and separated from each other. By using this detection is possible and count function works properly.

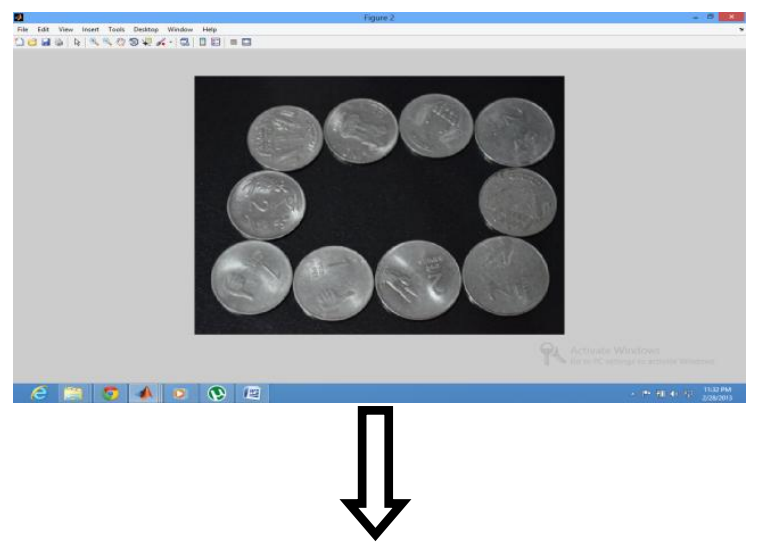



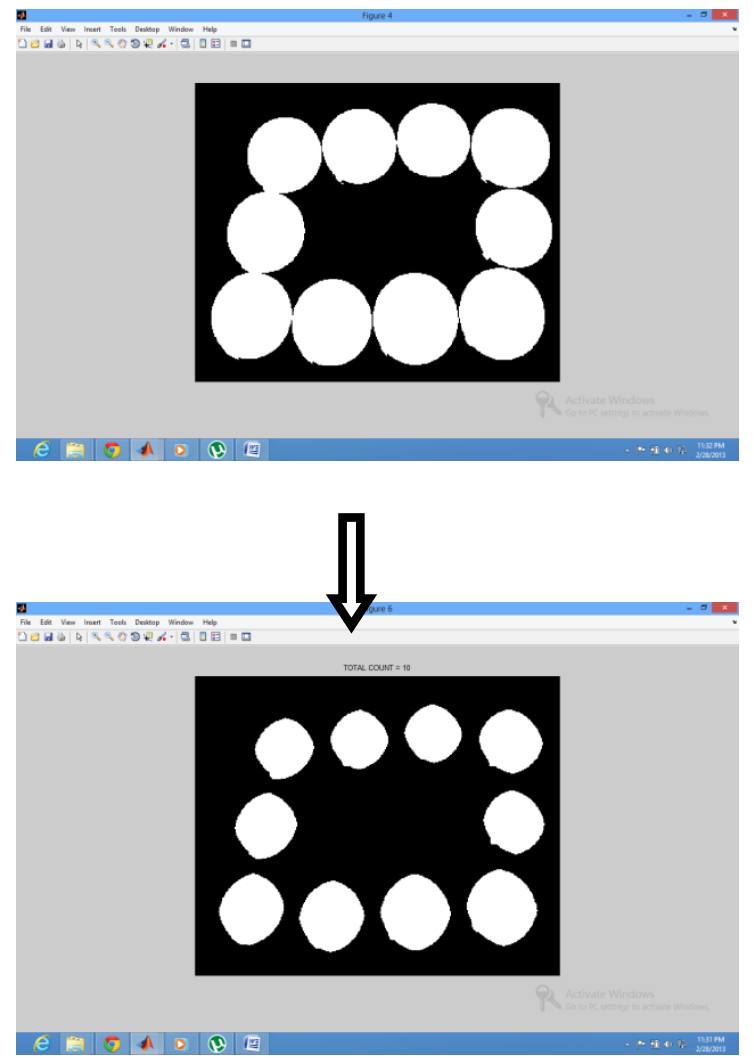

Figure (6): Coins placed touching each other

In figure (7), the same procedure is followed that is followed on figure (6), i.e. original image to binary image then this binary image to "erode" operation and it is failed, because coins are partially overlapped with each other. So, its detection is not done. If the value of "erode" operation is increased the coins start separating but they start diminish.

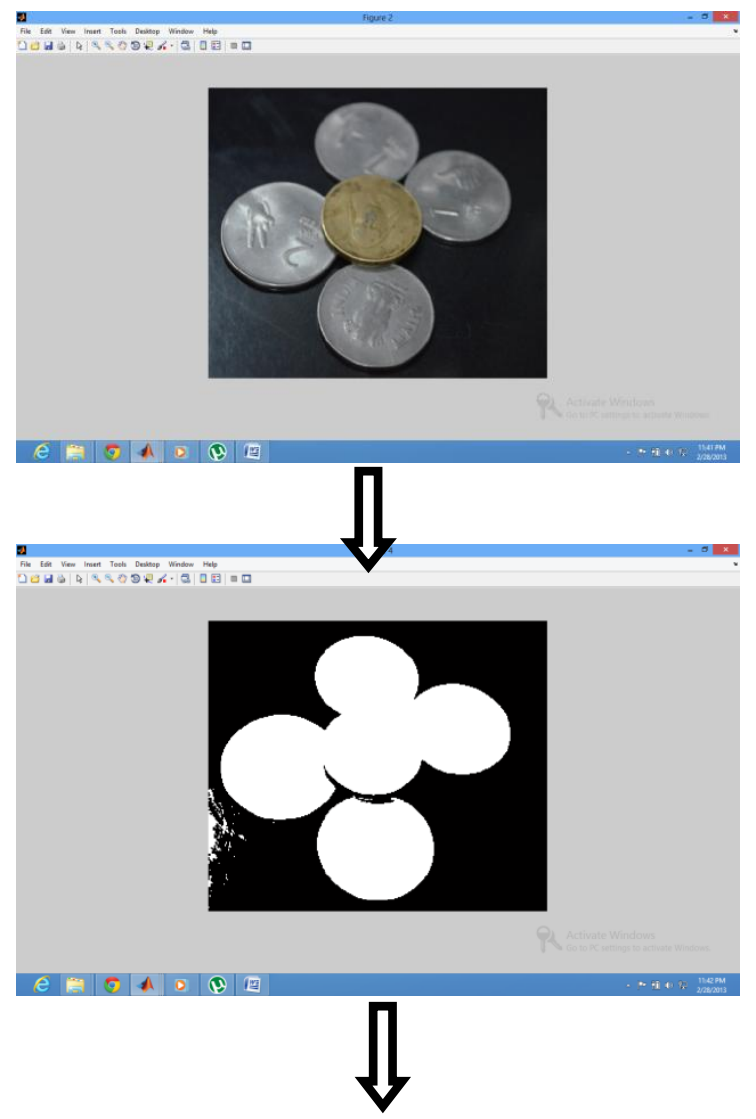

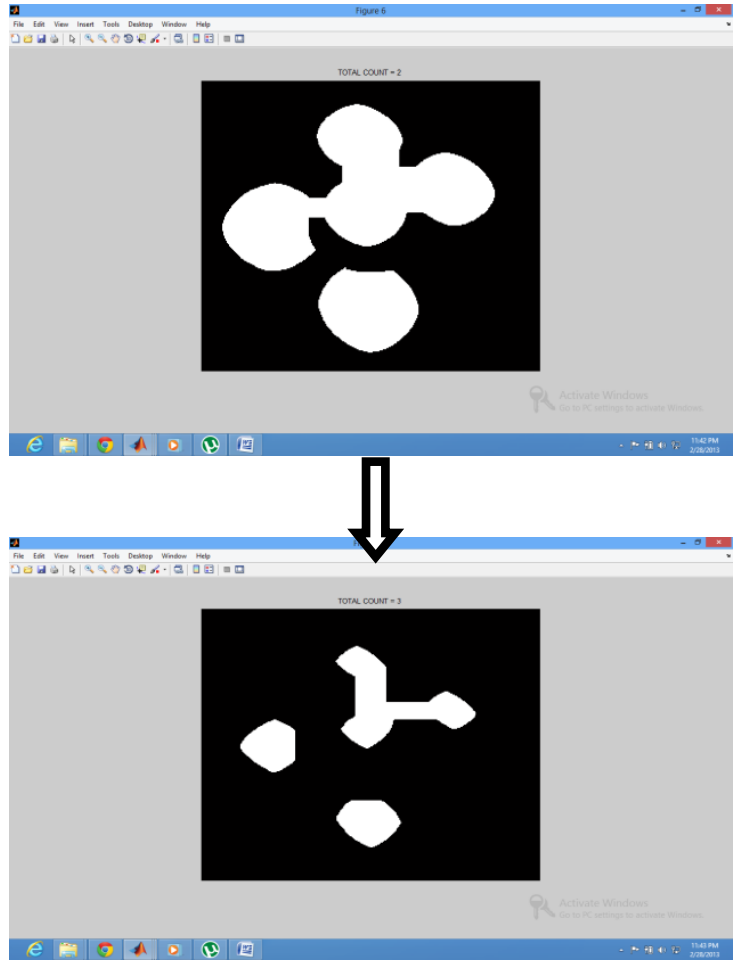

Figure (7): Partially overlapped coins

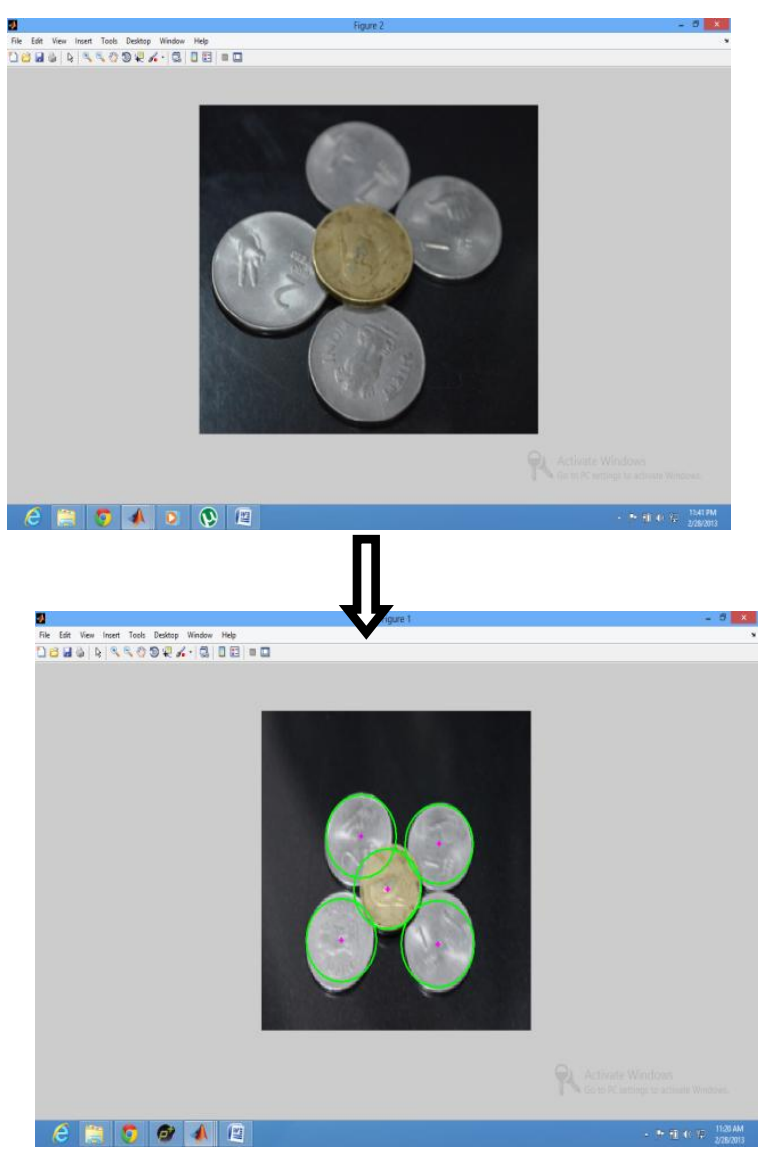

Figure(8): Partial Overlapped Coins

In figure(8), partial overlapped coins are taken. The morphing operation is failed in this case. So, to overcome this problem CHT is applied in which radius is calculated according to 
edges and accumulator space. This process is very efficient to work with partially overlapped coins.

This technique works efficiently with any type of partially overlapped coins as shown in figure (9) and in figure (10).

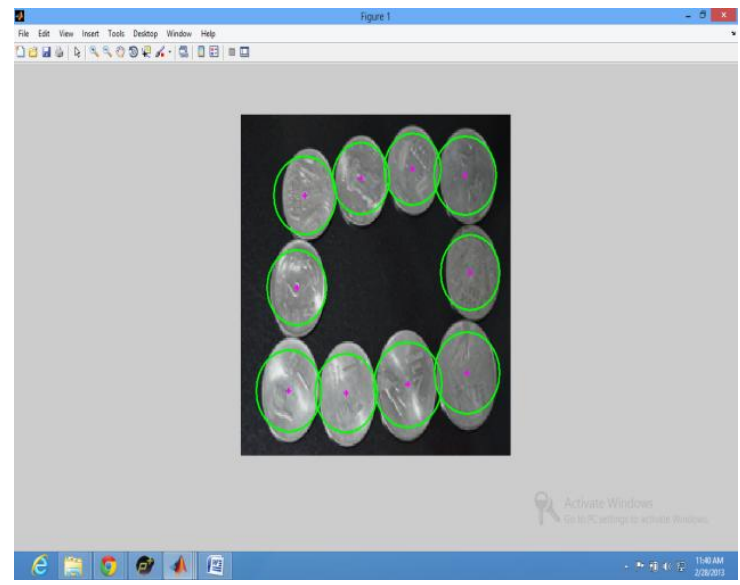

Figure(9): Detection of coins touching each other using CHT.

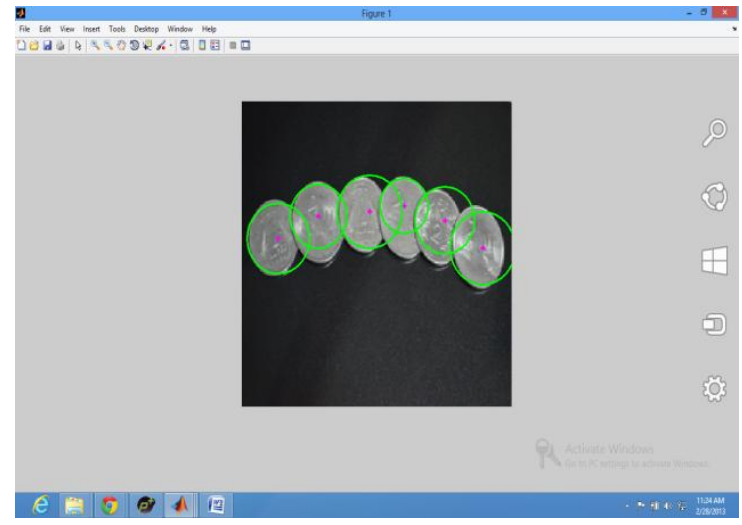

Figure (10): Detection of partially overlapped coins using CHT.

\section{CONCLUSION}

The radius of the coin is used to find out different coins. When the coin is put on the plain surface the accuracy is finding out in the edge detection as no floss edge are found.
For smooth edges canny edge detection is used as it give thin edges. This will help the CHT to work smoothly.

The problem faced by the recognition is when image is taken from far away and image is too small to work upon. The work can be further improved by detection of coins which are totally overlapped with each other.

\section{ACKNOLEGEMENT}

In all humility, I owe my deep and sincere gratitude to Lovely Professional University, CSE department, Jalandhar India for the continuous encouragement, estimated supervision and paternal affection throughout the period of this research. The improvements in this research work should not be possible without the guidance and suggestion of my guide.

\section{REFERENCES}

[1] Rafael C. Gonzalez, Richard E. Woods, Steven L. Eddins, Digital Image Processing using MATLAB, second edition.

[2] RadoslavFasuga, Petr Kasper, Martin Surkovsky, "Utilization of an Image Specific Areas in Searching", 2010 Fourth International Conference on Digital Society.

[3] C.M. VELU and P.VIVEKANANDA, "Indian Coin Recognition System of ImageSegmentation by Heuristic Approach and Hough Transform (HT)", Int. J. Open Problems Compt. Math., Vol. 2, No. 2, June 2009.

[4] Dr.Neelu Jain and NehaJain(2012) "Coin recognition using Circular Hough Transform", International Journal of Electronics Communication and Computer Technology(IJECCT), Volume 2 Issue 3(May 2012).

[5] RadoslavFasuga, Petr Kasper, Martin Surkovsky, "Utilization of an Image Specific Areas in Searching", 2010 Fourth International Conference on Digital Society.

[6] http://cmm.ensmp.fr/

[7] Mohamed Rizon, H. Y. (2005). Object Detection using Circular Hough Transform. American Journal of Applied Sciences, ISSN 1546-9239. 